MADELLA-OLIVEIRA, A.F., QUIRINO, C.R. e PACHECO, A. Principais hormônios que controlam o comportamento reprodutivo e social das fêmeas ruminantes - Revisão. PUBVET, Londrina, V. 8, N. 3, Ed. 252, Art. 1668, Fevereiro, 2014.

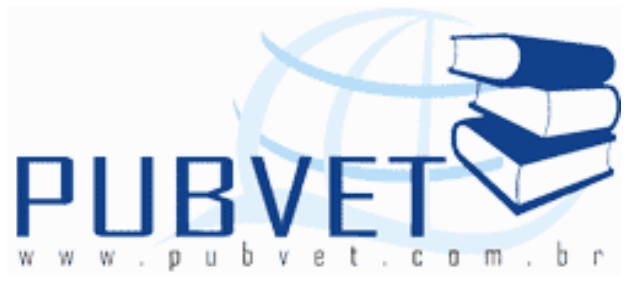

PUBVET, Publicações em Medicina Veterinária e Zootecnia.

\title{
Principais hormônios que controlam o comportamento reprodutivo e social das fêmeas ruminantes - Revisão
}

Aparecida de Fátima Madella-Oliveira ${ }^{1}$, Celia Raquel Quirino ${ }^{2}$, Aline Pacheco ${ }^{3}$

${ }^{1}$ Professora do Instituto Federal de Educação, Ciência e Tecnologia do Espírito Santo, Alegre, ES, Brasil. amadella@ufes.edu.br

2 Professora da Universidade Estadual do Norte Fluminense Darcy Ribeiro Centro de Ciências e Tecnologias Agropecuárias Campos dos Goytacazes, RJ, Brasil. crq@uenf.br

3 Pós-doutoranda da Universidade Estadual do Norte Fluminense Darcy Ribeiro - Centro de Ciências e Tecnologias Agropecuárias Campos dos Goytacazes, RJ, Brasil. apvuff@yahoo.com.br

\section{Resumo}

Esta revisão de literatura tem por objetivo identificar os principais hormônios que controlam o comportamento reprodutivo e social das fêmeas ruminantes, em primeiro lugar serão abordadas as definições, as classificações e as origens dos hormônios, em segundo lugar focalizará as principais funções dos hormônios no ciclo estral das fêmeas ruminantes, terceiro lugar caracterizarse-á os aspectos do comportamento social e por último serão relacionados alguns hormônios que causam alterações no comportamento reprodutivo e social. São vários os estudos sobre os hormônios que controlam o 
MADELLA-OLIVEIRA, A.F., QUIRINO, C.R. e PACHECO, A. Principais hormônios que controlam o comportamento reprodutivo e social das fêmeas ruminantes - Revisão. PUBVET, Londrina, V. 8, N. 3, Ed. 252, Art. 1668, Fevereiro, 2014.

comportamento reprodutivo em fêmeas ruminantes. Entretanto, poucos hormônios foram pesquisados em relação ao comportamento social.

Palavras-chave: Búfalos, Caprinos, Ciclo estral, Fisiologia, Hierarquia social, Ovinos.

\title{
Main hormones that control the reproductive and social behavior of female ruminants - Review
}

\begin{abstract}
This literature review aims to identify the main hormones that control the reproductive and social behavior of female ruminants, first we will discuss the definitions, classifications and origins of hormones, second will focus on the main functions of hormones in estrous cycle females ruminants, Third aspects of social behavior and finally some hormones that cause changes in the reproductive and social behavior will be related characterize herself. Several studies on the hormones that control the reproductive behavior in female ruminants exist. However, few hormones were studied in relation to social behavior.
\end{abstract}

Keywords: Buffaloes, goats, estrous cycle, physiology, social hierarchy, sheep.

\section{Introdução}

Trabalhos recentes sobre o Comportamento Animal têm demonstrado a influência do comportamento e da organização social sobre os processos fisiológicos e celulares. Variações no ambiente social podem inibir ou estimular ovulação. Outros estudos mostram que a qualidade do ambiente social e comportamental tem efeito direto sobre o funcionamento do sistema imunológico. Pesquisadores em Fisiologia e Imunologia necessitam ser orientados por estas influências comportamentais e sociais para garantir um controle adequado de seus próprios estudos (SNOWDON, 1999). 
MADELLA-OLIVEIRA, A.F., QUIRINO, C.R. e PACHECO, A. Principais hormônios que controlam o comportamento reprodutivo e social das fêmeas ruminantes - Revisão. PUBVET, Londrina, V. 8, N. 3, Ed. 252, Art. 1668, Fevereiro, 2014.

É inquestionável que grande parte do comportamento das espécies é possível graças à presença ou ausência de hormônios em determinados pontos do corpo. A disposição de um animal pode ser determinada por hormônios específicos que circulam o corpo, tornando o animal particularmente sensível a determinados estímulos ambientais. Desta forma, os estímulos que evocam o comportamento da reprodução têm maior probabilidade de desencadear a atividade apropriada em certas épocas; noutras, na ausência desses hormônios, o comportamento de reprodução apresenta menor probabilidade de ocorrência, mesmo na presença de estímulos adequados (CARTHY, 1980).

Esta revisão tem por objetivo identificar os principais hormônios que controlam o comportamento reprodutivo e social das fêmeas ruminantes.

\section{Definição, classificação e origem dos hormônios}

Algumas definições clássicas de hormônios podem ser encontradas na literatura. Os hormônios são substâncias químicas liberadas no sangue em pequenas quantidades e transportadas pelo sistema circulatório por todo o corpo até a célula alvo distantes, iniciando as respostas fisiológicas (CONSTANZO, 1999, HICKMAN et al., 2004). Os hormônios são sintetizados e secretados por células endócrinas, encontradas, geralmente, nas glândulas endócrinas (CONSTANZO, 1999). Segundo SENGER (2003) o hormônio é uma substância produzida em resposta a uma causa. O hormônio é uma substância produzida por uma glândula que age em um tecido distante (tecido alvo) e provoca uma mudança no tecido alvo. Essas mudanças podem envolver alterações no metabolismo, na atividade de síntese e de secreção. Assim, HAFEZ et al. (2004) mencionaram que os hormônios têm ação básica de inibir, estimular ou regular a atividade funcional de seus órgãos ou tecido-alvo.

Quimicamente, os hormônios são classificados como esteróides, peptídeos ou proteínas e aminas. Os hormônios esteróides são derivados do colesterol; os hormônios peptídeos ou protéicos são sintetizados a partir de 
MADELLA-OLIVEIRA, A.F., QUIRINO, C.R. e PACHECO, A. Principais hormônios que controlam o comportamento reprodutivo e social das fêmeas ruminantes - Revisão. PUBVET, Londrina, V. 8, N. 3, Ed. 252, Art. 1668, Fevereiro, 2014.

aminoácidos e os hormônios amina são derivados da tirosina (CONSTANZO, 1999).

Os hormônios esteróides são sintetizados e secretados pelo córtex suprarenal (adrenal), pelas gônadas e pela placenta. Os hormônios esteróides são o cortisol, aldosterona, estradiol e estriol, progesterona, testosterona e 1,25 diidroxicolecalciferol (CONSTANZO, 1999).

A maioria dos hormônios é de natureza peptídica ou protéica, podem-se citar alguns exemplos: hormônio liberador de gonadotrofina ( $\mathrm{GnRH})$, hormônio folículo-estimulante (FSH), hormônio luteinizante (LH), hormônio adrenocorticotrópico $(\mathrm{ACTH})$, ocitocina, melatonina, prolactina e leptina (CONSTANZO, 1999, HAFEZ et al., 2004).

Os hormônios aminados são catecolaminas (epinefrina, norepinefrina e dopamina) e os hormônios tiróideos (CONSTANZO, 1999).

Os hormônios hipotalâmicos são produzidos pelos neurônios no hipotálamo são hormônios liberadores de gonadotrofina (SENGER, 2003).

Os hormônios da hipófise dos lobos anteriores e posteriores são liberados dentro dos vasos sanguíneos. Os hormônios primários da reprodução liberados do lobo anterior da hipófise são hormônios do folículo-estimulante (FSH), hormônio luteinizante (LH) e a prolactina. E a ocitocina e liberada do lobo posterior da hipófise (SENGER, 2003, HAFEZ et al., 2004).

Os hormônios gonadais são originados das gônadas e afetam a função do hipotálamo, do lobo anterior da hipófise e os tecidos no processo reprodutivo. Os hormônios gonadais também inibem as características secundárias sexuais. Nas fêmeas, o ovário produz estrógenos, progesterona, inibina, alguma testosterona, ocitocina e relaxina (SENGER, 2003, HAFEZ et al., 2004).

Os hormônios também podem ser produzidos no útero e na placenta. Estes hormônios têm a função de manter a prenhez. São exemplos de hormônio uterino a prostaglandina $F_{2 a}$ e os hormônios da placenta incluem a progesterona, estrógenos, gonadotrofina coriônica eqüina (eCG) e gonadotrofina coriônica humana (hCG) (SENGER, 2003, HAFEZ et al., 2004). 
MADELLA-OLIVEIRA, A.F., QUIRINO, C.R. e PACHECO, A. Principais hormônios que controlam o comportamento reprodutivo e social das fêmeas ruminantes - Revisão. PUBVET, Londrina, V. 8, N. 3, Ed. 252, Art. 1668, Fevereiro, 2014.

\section{Principais funções dos hormônios no ciclo estral das fêmeas ruminantes}

O ciclo estral é regulado por mecanismos endócrinos e neuroendócrinos, principalmente os hormônios hipotalâmicos, as gonadotrofinas e os esteróides secretados pelos ovários. A regulação da secreção de gonadotrofina durante o ciclo estral requer um esmerado balanceamento entre complexas interações hormonais (HAFEZ e HAFEZ, 2004). Um componente conhecido pela sua importante influência é o hormônio liberador de gonadotrofinas (GnRH).

O GnRH secretado pelo hipotálamo, através do sistema portahipotalâmico-hipofisário, atinge esta glândula e estimula a liberação das gonadotrofinas, FSH (hormônio do folículo estimulante) e LH (hormônio luteinizante). Em seguida, o FSH e o LH atuam sobre os ovários, para estimular o desenvolvimento folicular, a ovulação e a síntese dos hormônios esteróides sexuais da fêmea (CONSTANZO, 1999).

Três esteróides são produzidos pelo ovário durante o ciclo estral: progesterona, estradiol e androstenediona. A progesterona é secretada exclusivamente pelo corpo lúteo, o estradiol pelos folículos e a androstenediona é produzida pelo corpo lúteo, pelos folículos e pelo estroma ovárico. O estradiol e a progesterona estão ligados à manifestação do cio e manutenção da gestação (GONZÁLEZ, 2002).

$O$ ciclo estral das fêmeas de ruminantes pode ser dividido em duas fases distintas. A primeira: fase folicular, que é iniciada após a luteólise que resulta no declínio da progesterona (SENGER, 2003). O período do desenvolvimento folicular pode ser dividido em proestro e estro. Assim, a fase folicular consiste em quatro grandes eventos. São eles: 1) liberação da gonadotrofina do lobo anterior da hipófise; 2) crescimento folicular e preparação da ovulação; 3) receptividade sexual e 4) ovulação (SENGER, 2003). No período do proestro, a liberação do $\mathrm{GnRH}$ pelo hipotálamo estimula a secreção de FSH e LH da glândula pituitária. Os elevados níveis de FSH no sangue induzem o desenvolvimento dos folículos e, em sinergismo com $\mathrm{LH}$, estimulam a sua 
MADELLA-OLIVEIRA, A.F., QUIRINO, C.R. e PACHECO, A. Principais hormônios que controlam o comportamento reprodutivo e social das fêmeas ruminantes - Revisão. PUBVET, Londrina, V. 8, N. 3, Ed. 252, Art. 1668, Fevereiro, 2014.

maturação. À medida que o folículo se desenvolve, aumenta a produção de estradiol pelos folículos, e após uma determinada concentração, o estradiol estimula a manifestação do cio e a liberação maciça do LH, dando inicio a segunda fase (SENGER, 2003; HAFEZ E HAFEZ, 2004)

No período de estro, a ocorrência de elevados níveis de estradiol, além de induzirem a manifestação do cio, são responsáveis pela dilatação da cérvice, síntese de secreção do muco da vagina e o transporte dos espermatozóides no trato reprodutivo da fêmea (HAFEZ e HAFEZ, 2004).

A fase folicular, desde a regressão do corpo lúteo até a ovulação, é relativamente curta: 2 a 3 dias em ovelhas e cabras e 3 a 6 dias em vacas (HAFEZ e HAFEZ, 2004). Entretanto, GONZÁLEZ (2002) relata que a fase folicular dura em média, 2 dias na ovelha e 4 a 5 dias na vacas. Em relação às cabras da raça Saanen foram observados uma duração de 4 dias, segundo os autores GINTHER e KOT, (1994) e RAWLINGS et al. (2003). Em búfalas, segundo DEL REI (1988) a duração do proestro é 21,2 horas e o estro de 28 horas. Entretanto, BARUSELLI (1992) encontrou média para o proestro de $40,0 \pm 23,92$ horas e a duração do estro apresentou média de $14,78 \pm 7,36$ horas em búfalas no Vale do Ribeira.

A segunda é a fase luteal, após o término da manifestação do cio, tem inicio o período de desenvolvimento do corpo lúteo (SENGER, 2003, HAFEZ e HAFEZ, 2004). A fase luteal é dividida em metaestro e diestro. A fase luteal consiste em três grandes processos. São eles: 1) a transformação das células foliculares em células luteal após ovulação (luteinização); 2) crescimento e desenvolvimento do corpo lúteo que produz em alta quantidade de progesterona (diestro); 3) destruição do corpo lúteo (luteólise) resultando a na subseqüente fase folicular (SENGER, 2003).

O corpo lúteo é um órgão temporário que funciona durante o diestro nos animais ciclantes ou durante a gestação. O corpo lúteo secreta a progesterona, hormônio responsável pela preparação do útero para a manutenção da gestação. Os níveis de progesterona plasmática são virtualmente indetectáveis logo depois da ovulação, mas começam a aumentar gradualmente a partir do 
MADELLA-OLIVEIRA, A.F., QUIRINO, C.R. e PACHECO, A. Principais hormônios que controlam o comportamento reprodutivo e social das fêmeas ruminantes - Revisão. PUBVET, Londrina, V. 8, N. 3, Ed. 252, Art. 1668, Fevereiro, 2014.

terceiro dia do ciclo mantendo-se elevados durante 12 dias na maioria das espécies. Os altos níveis de progesterona mantêm baixos os níveis de gonadotrofinas, acabando a vida do corpo lúteo, os níveis de progesterona voltam a cair a níveis indetectáveis (GONZÁLEZ, 2002).

A função do corpo lúteo está regulada por três sistemas, a - a hipófise que secreta LH é responsável pela regulação primária da secreção de progesterona como principal fator luteotrófico (GONZALEZ, 2002); b - útero que produz o fator luteolítico, a prostaglandina $F_{2 a}\left(P F_{2 a}\right)$ encontrado em várias espécies de mamíferos, exceto nos primatas. A PGF $F_{2 a}$ uterina controla a vida útil do corpo lúteo, que, em contrapartida, regula a extensão do ciclo. Ocorrendo a gestação, a influência luteolítica uterina é anulada, pois a progesterona secretada pelo corpo lúteo é necessária para a manutenção da gestação (GONZALEZ, 2002, HAFEZ e HAFEZ, 2004); e c - o conceptus, que tem efeito luteotrópico (GONZÁLEZ, 2002).

A luteólise ocorre sempre que não houver um embrião viável no útero no dia 11 a 12 do ciclo nas ovelhas e 16 e 17 dias na vaca (GONZÁLEZ, 2002). Segundo HAFEZ e HAFEZ (2004) a fase luteal em ovelhas tem uma duração é 14 a 15 dias e nas vacas é de 16 a 17 dias. GINTHER e KOT (1994) encontram duração média de 17 dias para cabras da raça Saanen. Em novilhas bubalinas o metaestro tem duração de 19 horas e diestro de 17 dias e 10 horas (DEL REI, 1988). BARUSELLI (1992) apresentou valores de média para a fase luteal

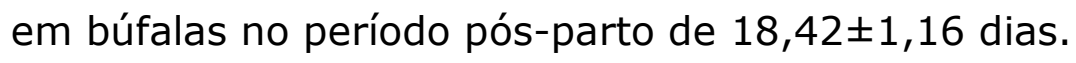

\section{Aspectos do comportamento social}

Em um sentido geral, qualquer interação que resulte da resposta de um animal a outro da mesma espécie representa comportamento social. A interação é apenas um acontecimento breve no tempo. A descrição de uma interação implica a presença e a identificação no mínimo de dois indivíduos; a descrição do conteúdo da interação, isto é, o indicador e o receptor da 
MADELLA-OLIVEIRA, A.F., QUIRINO, C.R. e PACHECO, A. Principais hormônios que controlam o comportamento reprodutivo e social das fêmeas ruminantes - Revisão. PUBVET, Londrina, V. 8, N. 3, Ed. 252, Art. 1668, Fevereiro, 2014.

interação, o tipo de interação: sexual, agressiva, cuidado parental, etc. (LE PENDU, 2004).

Segundo BOUISSOU et al. (2001) a interação social pode ser dividida em agonística (incluindo a agressividade, ações e respostas para a agressão, principalmente reações de se afastar) e não-agonística (inclui em particular o comportamento da prole e comportamento sexual).

O conjunto das interações entre dois indivíduos é uma relação social. Ao contrário de uma interação, uma relação social pode estender-se durante um longo período (LE PENDU, 2004).

Para muitas espécies, a coesão do grupo é crucial para o bem-estar do animal. Os rebanhos bovinos funcionam como uma unidade, e cada membro do grupo têm uma relação social especial com todos os outros. Cada membro conhece sua posição na hierarquia do grupo, e a aceitação deste fato tem vantagem de reduzir os encontros agressivos. Podem acontecer muitas brigas, no entanto, antes que a ordem hierárquica seja estabelecida (KLEMM, 1996).

A dominância se estabelece nesses grupos pela competição, ou seja, ela é produto de interações agressivas entre os animais do mesmo grupo ao competirem por um determinado recurso, definindo quem terá prioridade no acesso à comida, à água, à sombra, etc. O dominante é o indivíduo ou indivíduos do grupo que ocupam as posições mais altas na hierarquia, dominam os demais os atacando impunemente e têm prioridade em qualquer competição; os submissos (ou dominados) são os que se submetem aos dominantes. Os fatores que normalmente determinam à posição na hierarquia são: o peso, a idade e a raça (PARANHOS DA COSTA, 2002).

A dominância não é uma condição constante, e provavelmente a habilidade para ser dominante é em função do peso e das condições físicas mas isso não é sempre ao acaso (Klopfer e Hailman, 1967, citado por TULLOCH, 1992). A dominância e subordinação ocorrem juntas. Assim o comportamento pode ser descrito como agonístico: um animal ameaça ou luta enquanto os outros estão passivos ou tentam escapar (TULLOCH, 1992). 
MADELLA-OLIVEIRA, A.F., QUIRINO, C.R. e PACHECO, A. Principais hormônios que controlam o comportamento reprodutivo e social das fêmeas ruminantes - Revisão. PUBVET, Londrina, V. 8, N. 3, Ed. 252, Art. 1668, Fevereiro, 2014.

\section{Alguns hormônios que causam alterações no comportamento reprodutivo e social}

\subsection{Hormônio liberador de gonadotrofina (GnRH)}

O GnRH é um decapeptídeo com peso molecular de 1.183 dáltons. Ele é sintetizado e armazenado no hipotálamo basal médio. O GnRH fornece uma ligação humoral entre os sistemas endócrino e nervoso. Em resposta à estimulação nervosa, pulsos de GnRH são liberados no sistema portahipotálamo-hipofisário promovendo a liberação de LH e FSH da hipófise anterior (HAFEZ et al., 2004). Assim, promovendo o comportamento reprodutivo (DELLOVADE et al., 1998). O GnRH é largamente responsável pela iniciação do comportamento sexual, GnRH ativa uma cascata fisiológica que causa o desenvolvimento das gônadas (BENTLEY et al., 2006).

A secreção dos hormônios liberadores hipotalâmicos é modulada pelos níveis dos hormônios secretados nos órgãos-alvo primários e secundários. $O$ controle da secreção do $\mathrm{GnRH}$ é feito pelas próprias gonadotrofinas hipófisárias (LH e FSH) e pela progesterona e o estradiol (GONZÁLEZ, 2002).

Segundo HOFMANN (2006) apesar do conhecimento molecular crescente, sabe-se muito pouco sobre a sinalização do $\mathrm{GnRH}$ em relação às influencias ambientais e o comportamento reprodutivo. CARATY et al. (2002) demonstraram pela primeira vez que GnRH está envolvido no controle da receptividade de uma das espécies de ruminantes. O GnRH pode manter e estender o comportamento de receptividade nas ovelhas, mesmo depois que E2 desaparecesse da circulação periférica.

Vacas tratadas com antagonista do GnRH durante o desenvolvimento da fase luteal, prejudica a função normal do corpo lúteo, indicando que é necessário a liberação pulsatil do LH. Entretanto, se este tratamento for feito depois do desenvolvimento do corpo lúteo não tem nenhum efeito na secreção da progesterona. Em ovelhas tratadas com GnRH antagonista durante a fase luteal teve muito pequeno efeito na secreção de progesterona (NISWENDER et al., 2000). 
MADELLA-OLIVEIRA, A.F., QUIRINO, C.R. e PACHECO, A. Principais hormônios que controlam o comportamento reprodutivo e social das fêmeas ruminantes - Revisão. PUBVET, Londrina, V. 8, N. 3, Ed. 252, Art. 1668, Fevereiro, 2014.

Segundo DELLOVADE et al., (1998) os estímulos do comportamento social pode modificar os neurônios de GnRH em mamíferos. Contudo, de acordo com a literatura consultada não foram encontrados trabalhos sobre o controle do GnRH no comportamento social de ruminantes.

\subsection{Hormônio do Folículo-estimulante (FSH) e Hormônio luteinizante} (LH)

O hormônio do folículo-estimulante é um membro da família das glicoproteínas que tem papel central e essencial na reprodução. O FSH é fundamental na fisiologia da reprodução, regulando a fertilidade, estimulando o crescimento e a maturação do folículo ovariano ou folículo de De Graaf. O FSH por si só não causa secreção de estrógenos no ovário; ao contrário, ele necessita da presença do LH para estimular a produção estrogênica (ROSE et al., 2000, HAFEZ et al., 2004).

O hormônio luteinizante é uma glicoproteína composta de uma subunidade alfa e uma beta com o peso molecular de 30.000 dáltons e meiavida biológica de 30 minutos. Níveis tônicos ou basais de LH atuam em conjunto com o FSH no sentido de induzir a secreção de estrógenos do folículo ovariano desenvolvido. O pico pré-ovulatório do LH é responsável pela ruptura da parede folicular e ovulação (HAFEZ et al., 2004).

$\mathrm{O}$ LH ao longo do ciclo estral obedece a uma regulação feedback negativa em respostas a baixos níveis de estrógenos e progesterona e que se torna de regulação feedback positiva horas antes da ovulação, quando respondem a altos níveis de estrógenos (GONZÁLEZ, 2002).

De acordo com a literatura consultada não foram encontrados trabalhos sobre o efeito das gonadotrofinas no controle do comportamento social das fêmeas ruminantes. Entretanto, em aves foi observado que as gonadotrofinas controlam o comportamento de agressividade e pode ter efeito significado fora da estação reprodutiva (CROOK e BUTTERFIELD, 1968) 
MADELLA-OLIVEIRA, A.F., QUIRINO, C.R. e PACHECO, A. Principais hormônios que controlam o comportamento reprodutivo e social das fêmeas ruminantes - Revisão. PUBVET, Londrina, V. 8, N. 3, Ed. 252, Art. 1668, Fevereiro, 2014.

\subsection{Prolactina}

A prolactina é um hormônio polipeptídeo secretado pela adeno-hipófise. Ela é secretada dos terminais nervosos, predominantemente no núcleo arqueado localizado na eminência média, e transportada pelo sistema portahipotálamo-hipofisário até a adeno-hipófise. A prolactina está envolvida na mediação dos efeitos estacionais e lactacionais na reprodução em animais domésticos. Existe um hormônio inibitório denominado fator inibidor da prolactina (PIF) que regula a secreção de prolactina. O PIF é, provavelmente, a catecolamina, dopamina, uma amina de baixo peso molecular sintetizada a partir da L-tirosina (HAFEZ et al., 2004).

A prolactina é secretada com flutuações durante os diferentes estados do ciclo reprodutivo. Aumento da prolactina ocorre durante a ovulação e também durante a fase luteal do ciclo ovárico na vaca (GONZÁLEZ, 2002).

De acordo com a literatura consulta não foram encontrados trabalhos com prolactina em relação ao comportamento social de fêmeas ruminantes, entretanto, outras espécies, como: os macacos (SHIVELY, 1998), o homem (GERRA et al., 1998) e em porcos (PEDERSEN et al., 1993) foram encontrados estudos sobre o efeito da prolactina no comportamento social.

\subsection{Ocitocina (OT)}

A ocitocina é sintetizada no núcleo supra-óptico do hipotálamo e transportada em pequenas vesículas envoltas por uma membrana através dos axônios do nervo hipotálamo-hipofisário. Elas são armazenadas nos terminais nervosos próximos dos leitos capilares na neuro-hipófise até a sua liberação para a corrente circulatória (HAFEZ et al., 2004).

A OT também é produzida pelo corpo lúteo. Desempenha um importante papel no processo reprodutivo (GIMPL e FAHRENHOLZ, 2001). Durante a fase folicular do ciclo estral e durante estágios de gestação, a OT estimula as contrações uterinas, que facilitam o transporte do esperma para oviduto durante o cio (HAFEZ et al., 2004). A OT ovariana está envolvida na função luteínica. Ela age no endométrio induzindo a liberação de prostaglandina $F_{2 a}$ 
MADELLA-OLIVEIRA, A.F., QUIRINO, C.R. e PACHECO, A. Principais hormônios que controlam o comportamento reprodutivo e social das fêmeas ruminantes - Revisão. PUBVET, Londrina, V. 8, N. 3, Ed. 252, Art. 1668, Fevereiro, 2014.

$\left(\mathrm{PGF}_{2 \mathrm{a}}\right.$ ), que possui uma ação luteolítica (regressão do corpo lúteo) (HAFEZ et al., 2004).

Os níveis de OT têm variações entre as espécies durante o ciclo ovárico em vaca, ovelha e cabra. A concentração sanguínea de OT aumenta depois da regressão do corpo lúteo. Os estrógenos ovaricos estimulam a liberação de OT pituitária enquanto que a progesterona inibe. Têm sido encontrados outros fatores não reprodutivos, como o estresse e osmolaridade plasmática que afeta a liberação de OT (GONZÁLEZ, 2002). Na ovelha, a OT de origem ovarica (corpo lúteo) inicia a liberação de PGF $_{2 a}$ endometrial (GONZÁLEZ, 2002).

Na revisão de GIMPL e FAHRENHOLZ (2001) descreveram que a OT modula os reflexos neuroendócrinos, assim, tendo papel no estabelecimento do comportamento social em ratos. Entretanto, não existem estudos em relação à ação da OT no comportamento social das fêmeas ruminantes.

\subsection{Melatonina}

A melatonina ( $\mathrm{N}$-acetil-5-metoxitriptamina) é sintetizada pela glândula pineal. As células do parênquima pineal captam o aminoácido triptofano da circulação e o convertem em serotonina. Duas etapas do metabolismo da serotonina estão sob o controle neural. A primeira é a conversão da serotonina em $\mathrm{N}$-acetil-serotonina, que depois é convertida em melatonina. A segunda etapa envolve a enzima formadora de melatonina, hidroxiindol-O-metiltransferase (HIOMT) (HAFEZ et al., 2004).

A melatonina tem efeito no fotoperíodo e no controle hormonal da estacionalidade reprodutiva, que transforma a mensagem do fotoperíodo em mensagem química (HAFEZ et al., 2004). A melatonina esta relacionada com a regulação do ritmo circadiano e com as mudanças estacionais na atividade reprodutiva de mamíferos com ovinos e bovinos (SILVA et al., 2003).

A ação da melatonina no controle da função reprodutiva ocorre através da modulação da secreção de $\mathrm{LH}$, em que as alterações nos seus níveis e freqüência de pulsos caracterizam a estação reprodutiva e não reprodutiva (Karsh et al., 1984, citado por SASA, 2003). 
MADELLA-OLIVEIRA, A.F., QUIRINO, C.R. e PACHECO, A. Principais hormônios que controlam o comportamento reprodutivo e social das fêmeas ruminantes - Revisão. PUBVET, Londrina, V. 8, N. 3, Ed. 252, Art. 1668, Fevereiro, 2014.

A síntese e a secreção de melatonina encontram-se significativamente elevadas durante os períodos de dias curtos. Longos períodos diários de secreção elevada de melatonina provavelmente são responsáveis pela indução dos ciclos ovarianos em ovelhas. A melatonina exógena regula a atividade gonadal ovina, a administração contínua de melatonina induz a atividade reprodutiva em ovelhas acíclicas em meados do verão (HAFEZ et al., 2004).

A espécie bubalina e poliéstrica estacional de dia curto. A variação na concentração sangüínea de melatonina sinaliza a estação do ano para o sistema reprodutivo. O aumento na concentração plasmática de melatonina após o por do sol e menor em indivíduos menos sensíveis ao fotoperíodo (BASTIANETTO et al., 2005). A explicação dos mecanismos de ação da melatonina sobre o eixo reprodutivo ainda é desconhecida, em novilhas bubalinas, mas o fato de se encontrarem diferenças na concentração de melatonina plasmática na época de maior atividade reprodutiva sugere que a melatonina pode representar importante papel na regulação da reprodução dessa espécie (MATTOS et al., 2000).

Não existem relatos científicos na literatura consulta do efeito da melatonina no comportamento social de ruminantes.

\subsection{Leptina}

A leptina é uma proteína de $16 \mathrm{kDa}$ produto do gene obeso (ob), secretada como um hormônio dos adipócitos, tem sido proposta de ser o fator indefinível ligando status metabólico e reprodução. A leptina tem origem na placenta e principalmente no tecido adiposo e hormônios que agem na regulação do metabolismo energético (insulina e glicorticóides) (EHRHARDT et al., 2001, WILLIAMS et al., 2002). A leptina pode contribuir para a regulação do metabolismo energético, comportamento no consumo de alimentos e reprodução em muitas espécies de animais ruminantes ou não, pode também participar de importantes eventos inclusive a puberdade (WILLIAMS et al., 2002, CUNNINGHAM et al., 1999). 
MADELLA-OLIVEIRA, A.F., QUIRINO, C.R. e PACHECO, A. Principais hormônios que controlam o comportamento reprodutivo e social das fêmeas ruminantes - Revisão. PUBVET, Londrina, V. 8, N. 3, Ed. 252, Art. 1668, Fevereiro, 2014.

Estudos têm demonstrado que a leptina é o hormônio responsável por regular a liberação do hormônio liberador de gonadotrofinas (GnRH), refletindo o estado nutricional e as reservas energéticas é um hormônio derivado dos adipócitos. Foi demonstrado que, em baixas condições nutricionais, os níveis de leptina e gonadotrofinas estão relativamente baixos, sendo revertida às concentrações de hormônio folículo estimulante (FSH) e hormônio luteinizante (LH) após aplicação exógena desse hormônio. Há relevantes evidências de que a leptina é o sinal metabólico para inibir a atividade reprodutiva em condições nutricionais deficientes e reservas energéticas inadequadas (CUNNINGHAM et al., 1999)

A leptina parece ser o elo entre a condição nutricional e a reprodução, ela age centralmente no eixo hipotálamo-hipófise através de receptores e do neuropeptideo $\mathrm{Y}$ (NPY); perifericamente, a leptina tem efeito direto nas gônadas (LIEFERS et al., 2005, ZIEBA et al., 2005 WILLIAMS et al., 2002). Como as concentrações de leptina são proporcionais à gordura corporal, surgiu à hipótese que a leptina também pode atuar como um sinal para o sistema reprodutivo de que existe reserva energética suficiente para dar suporte a uma bem sucedida concepção e prenhez (HOSSNER, 1998).

Recentes estudos evidenciam que o gene de leptina é funcionalmente pertinente em vacas e ovelhas e pode contribuir para os eventos mais importantes da reprodução, inclusive a puberdade (WILLIAMS et al., 2002. ZIEBA et al., 2005).

A leptina tem importante papel na maturação sexual das novilhas que alcança a puberdade durante fim primavera e o começo do verão. Além, concentrações aumentadas de leptina no soro por mais de $30 \%$ no começo do inverno para o solstício de verão em vacas maduras, e também aumentou com mudanças significantes em adiposidade. Porém, foram observadas mudanças limitadas na leptina circulante durante o ciclo estral. Novilhas em curto período de jejum apresentaram reduções na expressão de gene de leptina e na leptina circulante, concomitante com declínios na freqüência dos pulsos de $\mathrm{LH}$, e nas concentrações de soro de insulina e IGF-1. Embora curto período de jejum em 
MADELLA-OLIVEIRA, A.F., QUIRINO, C.R. e PACHECO, A. Principais hormônios que controlam o comportamento reprodutivo e social das fêmeas ruminantes - Revisão. PUBVET, Londrina, V. 8, N. 3, Ed. 252, Art. 1668, Fevereiro, 2014.

vacas maduras com excelente condição corporal não tem efeitos na freqüência de pulsos de LH (WILLIAMS et al., 2002).

A leptina antagoniza diretamente o efeito estimulatório da insulina sobre a esteroideogênese das células granulosas e da teca nos ovários das vacas, causando redução na secreção de estradiol (SPICER, 2001, WILLIAMS et al., 2002). Em novilhas, os níveis de leptina aumentam linearmente desde 16 semanas até a primeira ovulação, atingindo a maturidade sexual com um ano de idade (WILLIAMS, 2002, ZIEBA et al., 2005).

De acordo com a literatura consultada não existem trabalhos científicos sobre o efeito da leptina no comportamento social de ruminantes. Entretanto, em aves os autores LÕHMUS e SUNDSTRÖM (2004) descreveram pela primeira vez que existe interação entre a leptina e o comportamento social dos animais.

\subsection{Hormônios glicocorticóides}

Os hormônios glicocorticóides têm uma grande influência em diferentes aspectos da função cerebral. Estes esteróides controlam certos aspectos do ritmo circadiano, as taxas de secreção de CRF, ACTH e cortisol que são altas de manhã cedo, mas baixas tarde e a noite (GUYTON e HALL, 1997).

O CRF é um importante neurotransmissor e mediador da resposta neuroendócrina, cardiovascular, autonômica e imunológica, desempenhando assim um papel fundamental na resposta adaptativa e comportamental que ocorre durante períodos de estresse (AYALA, 2002).

O ACTH condiciona a secreção de glicocorticóides (cortisol e corticosterona) pelo córtex da adrenal. Qualquer tipo de estresse que excite o hipotálamo desencadeará a ação neste eixo e a elevação rápida de glicocorticóides a fim de preparar o organismo para as reações de luta ou fuga. O cortisol exerce um feedback negativo sobre a liberação de ACTH, tanto em nível de hipotálamo como da hipófise. No hipotálamo, ele induz a redução do CRF e na hipófise, reduz a quantidade de ACTH em resposta ao CRF. (GUYTON e HALL, 1997). 
MADELLA-OLIVEIRA, A.F., QUIRINO, C.R. e PACHECO, A. Principais hormônios que controlam o comportamento reprodutivo e social das fêmeas ruminantes - Revisão. PUBVET, Londrina, V. 8, N. 3, Ed. 252, Art. 1668, Fevereiro, 2014.

Um animal submetido a uma situação estressante ativa quatro resposta biológicas principais para reagir: o comportamento, o sistema nervoso autônomo, o sistema nervoso neuroendócrino e imunológica (MOBERG, 1987).

A ativação do eixo hipotalâmico-hipofisário-adrenal (HHA) pelo estresse, gera uma cascata de mensagens hormonais que culminam em um aumento nas concentrações do cortisol plasmático. Em situações agudas o eixo HHA libera adrenalina, noradrenalina e catecolaminas que desencadeiam reações adrenérgicas imediatas, causando mudanças em quase todo o sistema endócrino. Em situações crônicas, o estimulo estressante leva a efeitos negativos sobre o crescimento, a engorda, a reprodução e a sanidade (COSTA e SILVA, 2003).

Avaliando as modificações do comportamento reprodutivo em relação ao cortisol. DOBSON et al. (2000) estudaram o desenvolvimento dos folículos ovariano de novilhas tratadas com hormônio adrenocorticotrofico (ACTH), observaram que as concentrações de cortisol aumentaram a partir de $24 \mathrm{~h}$ após o inicio do tratamento com ACTH. Os resultados deste experimento mostraram que as concentrações de cortisol e progesterona permaneceram elevados mesmo depois interrupção do ACTH e os pulsos e ondas de $\mathrm{LH}$ diminuíram durante o tratamento com ACTH, mas não do FSH.

As mudanças observadas em relação ao comportamento social foram que o cortisol influencia nos comportamento de hierarquia. SOLANO et al. (2004) fizeram um teste da seringa para observar a interação social em vacas Zebu e avaliaram a concentração de cortisol no plasma sanguíneo, verificaram que as vacas subordinadas adotaram uma estratégia passiva durante o teste que thes permitiu ter um controle melhor sobre o estresse, assim, apresentando uma baixa concentração de cortisol, enquanto que as vacas com maior dominância apresentaram níveis mais alto de cortisol.

Em outra pesquisa sobre comportamento social em novilhas ADEYEMO e HEATH (1982) fizeram o teste de separação das novilhas, (novilhas separadas das companheiras), com essa mudança na rotina dos animais, os autores observeram que o nível de cortisol no plasma aumentou. No estudo de 
MADELLA-OLIVEIRA, A.F., QUIRINO, C.R. e PACHECO, A. Principais hormônios que controlam o comportamento reprodutivo e social das fêmeas ruminantes - Revisão. PUBVET, Londrina, V. 8, N. 3, Ed. 252, Art. 1668, Fevereiro, 2014.

HASEGAWA et al. (1997) que avaliaram o comportamento social de vacas de primeira cria, que foram colocadas em um outro grupo de animais não familiar (reagrupadas), os autores observaram que o comportamento das subordinadas foi afetado com a diminuição da produção de leite e aumento dos níveis de concentração de cortisol, mas com as vacas dominantes não foi observado estas alterações. Entretanto, ENCARNAÇÃO (1983) observou também baixas concentrações do corticosteróide no sangue de animais dominantes e maiores níveis à medida que decaia a posição na escala social do rebanho, onde o último classificado se apresentou mais estressado.

\subsection{Estrógenos}

O estradiol é o estrógeno primário, sendo que a estrona e o estriol representam outros estrógenos metabolicamente ativos (HAFEZ et al., 2004). O estradiol é o estrógeno biologicamente ativo produzido pelo ovário apresentando controle, através do hipotálamo, sobre a liberação dos hormônios folículo-estimulante (FSH) e luteinizante (LH) (CONSTANZO, 1999).

Os estrógenos possuem várias funções no comportamento reprodutivo, entre elas podem ser citadas: a) a responsabilidade pelas contrações do miométrio no inicio do cio, b) $\circ 17 \beta$ estradiol aumentam o estimulo a secreção de prolactina por inibir fator inibidor (PIF), c) o estradiol produz mudanças típicas do estro no trato genital e no comportamento permitindo a receptividade sexual, d) os estrógenos ovarianos estimulam a liberação da ocitocina pituitária enquanto a progesterona inibe (GONZÁLEZ, 2002) e e) atua no SNC induzindo comportamento de cio na fêmea, entretanto, pequenas quantidades de progesterona, juntamente com o estrógeno, são necessárias para indução do cio em algumas espécies, tais como a ovelha e a vaca (HAFEZ et al., 2004).

Há, uma série de evidências indicam que o estradiol pode ter um papel central no mecanismo luteolítico. O mecanismo de ação do estradiol tem sido alvo de intensos estudos, mais ainda não foi elucidado. Os estrógenos nas 
MADELLA-OLIVEIRA, A.F., QUIRINO, C.R. e PACHECO, A. Principais hormônios que controlam o comportamento reprodutivo e social das fêmeas ruminantes - Revisão. PUBVET, Londrina, V. 8, N. 3, Ed. 252, Art. 1668, Fevereiro, 2014.

vacas têm ação luteolítica quando administrados no dia 13 do ciclo estral por causa elevação de PGF $2 a$ (GONZÁLEZ, 2002).

Em ovelhas, como em muitas outras espécies, as mudanças de comportamento e a secreção de LH pré ovulatório são ativadas com o aumento do E2 secretada pelo folículo em desenvolvimento no final da fase folicular. Em ovelhas uma injeção de $15 \mathrm{~g}$ de E2 é suficiente para uma ovelha com $50 \mathrm{~kg}$ exibir estro (CARATY et al., 2002).

Estudos de administração de hormônio em várias espécies (aves, ratos, camundongos e no homem) indicam que os estrógenos modulam agressão. Também há evidência que o comportamento social tem efeitos importantes na produção de estrógenos, que pode ter um importante papel no comportamento social desencadeando a agressividade (TRAINOR et al., 2006).

\subsection{Progesterona $\left(P_{4}\right)$}

A progesterona é um progestágeno natural de maior prevalência, sendo secretada pelas células luteínicas do corpo lúteo, pela placenta e glândulas adrenais. A progesterona é transportada na circulação sanguínea por uma proteína de ligação, globulina carreadora. Sua secreção é estimulada primariamente pelo LH (CONSTANZO, 1999, HAFEZ et al., 2004).

Entre as funções da progesterona, e em relação ao comportamento reprodutivo das fêmeas, ela atua sinergicamente com os estrógenos na indução do comportamento do cio, provoca inibição do cio e do pico préovulatório do LH quando em níveis elevados. A progesterona tem importante papel na regulação hormonal do ciclo estral (NISWENDER et al., 2000 e HAFEZ et al., 2004).

Em ovelhas, um dos fatores que controla o comportamento de estro é a P4, presente na fase luteal, na qual aumenta o número de receptores de E2, aumento os níveis de E2 (CARATY et al., 2002).

HOGAN et al. (2004) estudaram o comportamento social, sexual e avaliaram as medidas das concentrações de progesterona no leite das cabras de Nova Zelândia, não foi encontrada nenhuma alteração das concentrações de 
MADELLA-OLIVEIRA, A.F., QUIRINO, C.R. e PACHECO, A. Principais hormônios que controlam o comportamento reprodutivo e social das fêmeas ruminantes - Revisão. PUBVET, Londrina, V. 8, N. 3, Ed. 252, Art. 1668, Fevereiro, 2014.

progesterona, entretanto, ocorreram mudanças no comportamento das fêmeas em estro, contudo, estas mudanças pareciam não influenciar no estado reprodutivo do grupo.

\subsection{Testosterona}

A testosterona é um andrógeno produzido pelas células intersticiais, células de Leyding nos machos e nas fêmeas são produzidas nas células da teca (SENGER, 2003). A testosterona nas fêmeas passa para as células da granulosa, onde sob o efeito do FSH é transformada em estrógeno.

A testosterona também estimula a síntese e liberação da progesterona $\left(P_{4}\right)$ pelas células da granulosa em vacas (HAFEZ et al., 2004).

Existem várias pesquisas que relacionam o mecanismo de agressão com a testosterona, em várias espécies foram observados que os elevados níveis de testosterona aumentaram o comportamento de agressividade (TRAINOR et al., 2006). Entretanto, não seria precipitado concluir tal efeito, segundo BOUISSOU, (1978) quando se aplica injeções de andrógeno em vacas, ocorre modificação na estrutura social do grupo.

Em outro estudo BOUISSOU e GAUDIOSO (1982) avaliaram o comportamento social de novilhas com tratamento com andrógenos, e concluíram que os mecanismos pelos quais os andrógenos atuam não esta bem compreendida. Entretanto, para comportamento emocional e agressivo os andrógenos poderiam explicar o status social em ungulados.

\subsection{Prostaglandina}

As prostaglandinas podem ser consideradas hormônios que regulam diversos fenômenos fisiológicos e farmacológicos, tais como a contração da musculatura lisa dos tratos reprodutivo e gastrintestinal, a ereção, a ejaculação, o transporte espermático, a ovulação, a formação do corpo lúteo, o parto e a ejeção do leite. A secreção PGF $_{2 a}$ é controlada por três hormônios ovarianos: progesterona, estradiol e ocitocina (GONZÁLEZ, 2002). 
MADELLA-OLIVEIRA, A.F., QUIRINO, C.R. e PACHECO, A. Principais hormônios que controlam o comportamento reprodutivo e social das fêmeas ruminantes - Revisão. PUBVET, Londrina, V. 8, N. 3, Ed. 252, Art. 1668, Fevereiro, 2014.

As prostaglandinas estão ainda envolvidas na ovulação. Por exemplo, na ovelha e na vaca, a ovulação é bloqueada pela administração de indometacina, uma inibidora da síntese de prostaglandinas. Uma vez que a liberação de $\mathrm{LH}$ não é afetada nesses animais, a ação ao nível de folículo ovariano envolve a $\mathrm{PGF}_{2 a}$ e a $\mathrm{PGE}_{2}$ ou ambas (HAFEZ et al., 2004).

A regressão do corpo lúteo durante o ciclo estral depende de aumentos dos pulsos de $\mathrm{PGF}_{2 a}$. No período médio do ciclo estral os folículos secretam estradiol, que estimula a síntese de receptores de ocitocina no endométrio. 0 corpo lúteo secreta ocitocina que age sobre as células glandulares do endométrio e estimula a secreção de $\mathrm{PGF}_{2 a}$. Dessa forma segue-se a lise do corpo lúteo com a subseqüente queda dos níveis de progesterona. Ocorre a ovulação do folículo pré-ovulatório e se inicia um novo ciclo estral. Na fêmea prenha o número de receptores para ocitocina no endométrio são mais baixos e a liberação de $\mathrm{PGF}_{2 a}$ pelo endométrio se reduz em resposta a ação da ocitocina exógena, havendo a persistência do corpo lúteo, estrutura fundamental para a manutenção da gestação (GONZÁLEZ, 2002).

Em ovelhas, cabras e em vacas a $\mathrm{PGF}_{2 a}$ é um hormônio luteolítico liberado ciclicamente pelo útero tendo a ação no ovário e causa regressão no corpo lúteo (MCCRACKEN et al., 1999). Em outro experimento com ovelhas, injetando progesterona logo no inicio do ciclo e obtendo diminuição da duração do corpo lúteo de 12,3 dias para 8 dias. Ou seja, a liberação PGF $_{2 a}$ que normalmente ocorre no dia 11 a 12 do ciclo de ovelhas e no dia 16 a 17 nas vacas, reflete os altos níveis de progesterona nos primeiros dias da fase luteal do ciclo estral (GONZÁLEZ, 2002).

KABUGA et al., (1992) avaliaram o comportamento social de vacas durante $\mathrm{O}$ estro, tratadas com $\mathrm{PGF}_{2 \mathrm{a}}$, verificaram que o comportamento agonista (ameaças e brigas) não foi significativo enquanto que 0 comportamento não-agonistica aumentou significativamente. Observaram também que a atividade de monta e duração do estro, aumentaram significativamente. Em outra pesquisa CASTELLANOS et al., (1997) encontraram uma relação forte entre a estrutura social do rebanho e a 
MADELLA-OLIVEIRA, A.F., QUIRINO, C.R. e PACHECO, A. Principais hormônios que controlam o comportamento reprodutivo e social das fêmeas ruminantes - Revisão. PUBVET, Londrina, V. 8, N. 3, Ed. 252, Art. 1668, Fevereiro, 2014.

expressão do estro, com fêmeas tratadas com $\mathrm{PGF}_{2 \mathrm{a}}$. Os autores verificaram que o comportamento de monta durante o estro era mais alto em vacas do que em novilhas, e as vacas formaram grupos sociais mais estáveis que as novilhas.

\section{Considerações finais}

São vastos os estudos sobre os hormônios que atuam no comportamento reprodutivos das fêmeas de ruminantes. Entretanto, observa-se a escassez de pesquisas em relação aos hormônios que controlam o comportamento social nas fêmeas ruminantes, dentre os hormônios citados nesta revisão foram encontradas pesquisas que estão relacionadas com o cortisol, a progesterona, a testosterona e a prostaglandina. Assim, evidenciando que as pesquisas neste sentido ainda estão no estágio inicial.

\section{Literatura Citada}

ADEYEMO, O., HEATH, E. (1982) Social behaviour and adrenal cortical activity in heifers. Applied Animal Ethology, 8: 99-108.

AYALA, A. R. (2002) Antagonista do hormônio liberador de corticotrofina: Atualização e Perspectivas. Arq. Bras. Endocrinol Metab. 46 (6): 619-625

BARUSELLI, P. S. (1992) Atividade ovariana e comportamento reprodutivo no período pósparto em búfalos (Bubalus bubalis). Dissertação (Mestrado em Reprodução Animal) São Paulo, Faculdade de Medicina Veterinária e Zootecnia, Universidade de São Paulo, 99p.

BASTIANETTO, E., ESCRIVÃO, S. C., OLIVEIRA, D. A. A. (2005) Influência das características reprodutivas da búfala na produção, composição e qualidade do leite. Revista Brasileira de Reprodução Animal, 29: 49-52.

BENTLEY, G. E., JENSEN, J. P.,KAUR, G. J., WACKER, D. W., TSUTSUI, K., WINGFIELD, J. (2006) Rapid inhibition of female sexual behaviour by gonadotropin-inhibitory hormone (GnIH). Hormone and Behavior, Article in Press.

BOUISSOU, M. F. (1978) Effect of injections of testosterone propionate on dominance relationships in a group of cows. Hormones and Behavior, 11: 388-400.

BOUISSOU, M. F., GAUDIOSO, V. (1982) Effect of early androgen treatment on subsequent social behavior in heifers. Hormones and Behavior, 16: 132-146.

BOUISSOU, M. F., BOISSY, A., LE NEINDRE, P., VEISSIER, I. (2001) The social behaviour of cattle: Social behaviour in farm animals. eds: L.J. Keeling e H.W. Gonyou. Saint- GenésChampenelle, France. p. 113-145. 
CARATY, A., DELALEU, B. CHESNEAU, D., FABRE-NYS, C. (2002) Sequential role of E2 and GnRH for the expression of estrous behaviour in ewes. Endocrinology, 14: 139-145.

CARTHY, J.D. (1980) Hormônios e feromônios. Comportamento Animal. 2 ed. São Paulo: Editora Pedagógica Universitária Ltda e Editora da Universidade de São Paulo, p. 43-49.

CASTELLANOS, F., GALINA,C. S., ORIHUELA, J. A., NAVARRO-FIERRO, R., MONDRAGÓN, R. (1997) Estrous expression in dairy cows and heifers (Bos taurus) following repeated PGF2a injection and choice of selecting a mounting partner. Applied Animal Behaviour Science, 51, 29-37.

COSTA e SILVA, E. V. (2003) Ambiente e manejo reprodutivo: problemas e soluções. Anais do XIII Congresso Nacional de Zootecnia- Ambiência- Eficiência e qualidade na produção animal, Uberaba, MG, Brasil.

CONSTANZO, L. S. (1999). Fisiologia. 1 ed. Rio de Janeiro: Guanabara Koogan, 392p.

CROOK, J. H., BUTTERFIELD, P. A. (1968) Effects of testosterone propionate and luteinizing hormone on agonistic and nest building behaviour of Quelea quelea. Animal Behaviour, 16: 370-384.

CUNNINGHAM, M.J.; CLIFTON, D.K.; STEINER, R.A (1999) Leptin's actions on the reproductive axis: perspectives and mechanisms. Biology of Reproductive, 60: 216-222.

DEL REI, A. J.M. (1988) Contribuição ao estudo da eficiência reprodutiva na búfala - Bubalus bubalis Lin. Monografia (Produção de Ruminantes) Instituto de Zootecnia da Universidade Federal Rural do Rio de Janeiro. Rio de Janeiro. 38 p.

DELLOVADE, T., SCHWANZEL-FUKUDA, M., GORDAN, J., PFAFF, D. (1998) Aspects of GnRH. neurobiology conserved across vertebrate forms. General and Comparative Endocrinology, 112: 276-282.

DOBSON, H., RIBADU, Y., NOBLE, K.M., TEBBLE, J. E., WARD, W. R. (2000) Ultrasonography and hormone profiles of adrenocorticotrophic hormone (ACTH) - induced persistent ovarian follicles (cysts) in cattle. Journal of Reproduction and Fertility, 120: 405-410.

EHRHARDT, R. A., SLEPETIS, R. M., BELL, A. W., BOISCLAIR, Y. R (2001) Maternal leptin is elevated during pregnancy in sheep. Domestic Animal Endocrinology, 21: 85-96.

ENCARNAÇÃO, R.O. (1983) Estresse e produção animal. I. Crescimento, engorda qualidade de carne e carcaça. Ciência e Cultura, 35:773-777.

GERRA, G., ZAIMOVIC, A., GIUCASTRO, G., FOLLI, F., MAESTRI, D., TESSONI, A., AVANZINI, P., CACCAVARI, R., BERNASCONI, S., BRAMBILLA, F. (1998). Neurotransmitter-hormonal responses to psychological stress in peripubertal subjects: relationship to aggressive behaviour. Life Sciences, 62: 617-625.

GIMPL, G., FAHRENHOLZ, F., (2001) The oxytocin receptor system: structure, function, and regulation. Physiological Reviews, 81: 629-683.

GINTHER, O. J., KOT, K. (1994) Follicular dynamics during the ovulatory season in goats. Theriogenology, 42: 987-1001.

GONZÁLEZ, F.H. D. (2002) Introdução à endocrinologia reprodutiva veterinária. Copyright. Universidade Federal do Rio Grande do Sul. (Faculdade de Veterinária). 87p. 
GUYTON, A. C., HALL, J. E. (1997) Os hormônios Adrenocorticais. 9 ed. Rio de Janeiro: Guanabara Koogan, 1014p.

HAFEZ, E.S.E., HAFEZ, B. (2004). Ciclos reprodutivos: Reprodução Animal. 7 ed. São Paulo: Manole, p. 55- 67.

HAFEZ, E.S.E., JAINUDEEN, M. R., ROSNINA (2004) Hormônios, fatores de crescimento e reprodução. Reprodução Animal. 7 ed. São Paulo: Manole, p. 33-53.

HASEGAWA, N., NISHIWAKI, A., SUGAWARA, K., ITO, I. (1997) The effects of social Exchange between two groups of lactating primiparous heifers on milk production dominante order, behavior and adrenocortical response. Applied Animal Behaviour Science, 51: 15-27.

HICKMAN, C.P. J., ROBERTS, L.S., LARSON, A. (2004) Comportamento Animal: Princípios integrados de zoologia. 11. ed. Rio de Janeiro: Guanabara Koogan, p.741- 758.

HOFMANN, H. (2006) Gonadotropin-releasing hormone signalling in behavioural plasticity. Current Opinion in Neurobiology, Article in Press.

HOGAN, N., WASS, J. R., VERKERK, G. A. (2004) Can female-female stimulation of breeding condition occur in dairy goats? Small Ruminant Research, 55: 21-27.

HOSSNER, K. L. (1998) Cellular, molecular and physiological aspects of leptin: potential application in animal production. Canadian Journal of Animal Science, 78, 463-472.

KABUGA, J. D., GYAWU, P., ASARE, K., KARIKARI, P. K., APPIAH, P., AWUNYO, K., KWARTENG, A. (1992) Social behaviour at and around oestrus of N'dama cows treated with prostaglandin $F_{2 a}$ analogue, cloprostenol. Applied Animal Behaviour Science, 35: 103-114.

KLEMM, W. R. (1996) Fisiologia comportamental. In: Melvin J. Sewenson e Willian O. Reece (ed) Dukes-Fisiologia dos Animais Domésticos. 11a ed. Rio de Janeiro: Guanabara Koogan p.825-841.

LE PENDU, Y. (2004) Etologia Aplicada. Fundamentos de Etologia. http:// br.geocites.com/yvonnick01/curso_Etologia_aplicada.pdf. Acesso em 10 de novembro de 2004.

LIEFERS, S.C., VEERKAMP, R. F., TE PAS, M. F. W., CHILLIARD, Y., VAN DER LENDE, T. Genetics and physiology of leptin in periparturient dairy cows. Domestic Animal Endocrinology, 29: 227-238.

LÕHMUS, M., SUNDSTRÖN, L. F. (2004) Leptina and social environment influence the risktaking and feeding behaviour of Asian blue quail. Animal Behaviour, 68: 607-612.

McCRACKEN, J. A., CUSTER, E. E., LAMSA, J. C. (1999) Luteolysis: A neuroendocrine mediated-event. Physiological Reviews, 79: 263-323.

MATTOS, P.S. R., FRANZOLIN, R. NONAKA, K. O. (2000) Concentração plasmática de melatonina em novilhas bubalinas (Bubalus bubalis) ao longo do ano. Arquivo Brasileiro de Medicina Veterinária e Zootecnia, V(5) no. 5.

MOBERG, G. P. (1987) A model for assessing the impact $f$ behavioral stress on domestic animals. J. Anim. Sci. 65: $1228-1235$. 
NISWENDER, G. D.,JUENGEL, J. L., SILVA, P. J., ROLLYSON, M. K., McINTUSH, E. W. (2000) Mechanisms controlling the function and life span of the corpus luteum. Physiological Reviews, 80: 1-29.

PARANHOS DA COSTA, M. J. R. (2002) O comportamento social dos bovinos e uso do espaço. http://www.milkpoint.com.br. Acesso em 10 de outubro de 2004.

PEDERSEN, L. J., ROJKITTIKHUN, T., FINARSSON, S., EDQVIST, L. E. (1993) Postweaning grouped sows: effects of aggression on hormonal patterns and oestrous behaviour. Applied Animal Behaviour Science, 38: 25-39.

RAWLINGS, N. C., EVANS, A.C.O., HONARAMOOZ, A., BARTLEWSKI, P. M., (2003) Antral follicle growth and endocrine changes in prepubertal cattle, sheep and goats. Animal Reproduction Science, 78: 259-270.

ROSE, M. P., GAINeS, R. E., DAS, G., BALEN, A. H. (2000) Definition and measurement of follicle stimulating hormone. Endocrine Reviews, 21: 5-22.

SASA, A. (2003) Efeitos da nutrição na atividade ciclica reprodutiva e nas concentrações plasmáticas de melatonina em ovelhas mantidas em pastagem e submetidas ao efeito do macho durante 0 anestro sazonal. Tese (Mestrado em Qualidade e Produtividade Animal) Pirassununga - SP, Faculdade de Zootecnia e Engenharia de Alimentos da USP, 86p.

SENGER, P.L. (2003) Pathways to pregnancy and parturition. 2. ed. Washington: Current conceptions, 368p.

SHIVELY, C. A. (1998) Social subordination stress, behavior, and central monoaminergic function in female cynomolgus monkeys. Biological Psychiatry, 44: 882-891

SILVA, M.V. LOPES, P.S., GUIMARÃES, S. E., ALMEIDA TORRES, R. (2003). Utilização de marcadores genéticos em suínos. I. características reprodutivas e de resistência a doenças. Arch. Latinoam. Prod. Anim.11: 1-10.

SNOWDON, C.T. (1999) O significado da pesquisa em comportamento animal. Estudo da Psicologia, 4(2): 365-373.

SOLANO, J., GALINDO, F., ORIHUELA, A., GALINA, C. S. (2004) The effect of social rank on the physiological response during repeated stressful handling in Zebu cattle (Bos indicus). Physiology \& Behavior, 82: 679-683.

SPICER, L.J. (2001) Leptin: a possible metabolic signal affecting reproduction. Domestic Animal Endocrinology, 21: 251:270.

TRAINOR, B. C., KYOMEN, H. H., MARLER, C.A. (2006) Estrogenic encounters: how interactions between aromatase and the environment modulate aggression. Frontiers in Neuroendocrinology, Article in Press.

TULLOCH, D. G. (1992) Behaviour of non-domesticated swamp buffaloes in Australia. In: Tulloch, N. M., Holmes, J. H. G. (ed) Buffalo Production. Australia: Elsevier, p. 247-270.

WILLIAMS, G.L., AMSTALDEN, M., GARCIA, M.R., STANKO,R. L.,NIZIELSKI,E., MORRISON, C. D., KEISLER, D. H. (2002) Leptin and its role in the central regulation of reproduction in cattle. Domestic Animal Endocrinology, 23: 339-349. 
ZIEBA, D. A., AMSTALDEN, M., WILLIAMS, G. L. (2005) Regulatory roles of leptin in reproduction and metabolism: A comparative review. Domestic Animal Endocrinology, 29: 166185. 